\title{
TIC'S en canales de comercialización y distribución del sector carrocero de la provincia de Tungurahua
}

\section{TIC'S in the marketing and distribution channels of the bodybuilding sector of the province of Tungurahua}

1 Leslie Elizabeth Aguilera Criollo

https://orcid.org/0000-0002-4291-720X

Universidad Técnica de Ambato, Facultad de Ciencias Administrativas, Carrera de Marketing y Gestión de Negocios, Ambato, Ecuador

laguilera7292@uta.edu.ec

2 Carlos Avelino Bucay Pallango $\quad$ (iD) https://orcid.org/0000-0001-9404-7341

Universidad Técnica de Ambato, Facultad de Ciencias Administrativas, Carrera de Marketing y Gestión de Negocios, Ambato, Ecuador

cbucay0599@uta.edu.ec

3 Edwin César Santamaría Díaz (iD) https://orcid.org/0000-0003-2636-9685 Universidad Técnica de Ambato, Facultad de Ciencias Administrativas, Carrera de Marketing y Gestión de Negocios, Ambato, Ecuador ec.santamaria@uta.edu.ec

Artículo de Investigación Científica y Tecnológica Enviado: 15/09/2021

Revisado: 05/10/2021

Aceptado: 20/10/2021

Publicado:05/01/2022

DOI: $\underline{\text { https://doi.org/10.33262/cienciadigital.v6i1.1954 }}$

\section{Cítese:}

Aguilera Criollo, L. E., Bucay Pallango , C. A., \& Santamaría Díaz, E. C. (2022). TIC'S en canales de comercialización y distribución del sector carrocero de la

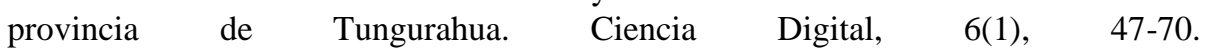
https://doi.org/10.33262/cienciadigital.v6i1.1954

CIENCIA DIGITAL, es una Revista multidisciplinaria, Trimestral, que se publicará en soporte electrónico tiene como misión contribuir a la formación de profesionales competentes con visión humanística y crítica que sean capaces de exponer sus resultados investigativos y científicos en la misma medida que se promueva mediante su intervención cambios positivos en la sociedad. https://cienciadigital.org

La revista es editada por la Editorial Ciencia Digital (Editorial de prestigio registrada en la Cámara Ecuatoriana de Libro con No de Afiliación 663) www.celibro.org.ec 
Palabras claves: TIC'S, comercialización, distribución, sector carrocero, sistemas

Keywords: TIC'S, commercialization, distribution, body builder sector, systems
Resumen

El sector carrocero en la provincia de Tungurahua es una de las principales actividades económicas a nivel nacional, estas facturan miles de dólares anualmente por la comercialización de sus bienes, por tanto, el éxito de este sector industrial depende del manejo óptimo de sus canales de comercialización y de distribución. Objetivo: Conocer el tipo de Tecnologías de la Información y Comunicación empleadas en los canales de comercialización y de distribución. Metodología: se tomó en consideración a 27 empresas registradas en la Cámara Nacional de Fabricantes de Carrocerías (CANFAC) y 10 empresas registradas en la Dirección de Producción de Tungurahua, para desarrollar la investigación se empleó el método descriptivo a través de una encuesta a los directivos de las empresas, misma que se validó con el índice de consistencia interna Alpha de Cronbach para variables ordinales, Kuder Richardson KR-20 para las preguntas dicotómicas y aprobación del instrumento por 5 jueces en esta caso se aplicó el coeficiente de confiabilidad V de Aiken, se utilizó la modalidad investigativa bibliográfica y de campo. Resultados: el sector industrial emplea medios electrónicos para sus canales de comercialización, el 68,9\% prefieren las redes sociales y las páginas web, el 79,1\% utilizan el canal directo para distribuir bienes, también existe un bajo conocimiento sobre el funcionamiento del sistema de planificación de recursos empresariales (ERP) y del sistema para la Administración de las relaciones con el cliente (CRM). Conclusión: las tecnologías de la información y comunicación poseen muchas alternativas, las mismas que son aplicadas dependiendo del tamaño que cada una posee, por tanto, sugerir alternativas que permitan gestionar de manera eficiente el canal de comercialización y de distribución en entre clientes internos y externos de la empresa.

\section{Abstract}

The bodybuilding sector in the province of Tungurahua is one of the main economic activities nationwide, they invoice thousands of dollars annually for the commercialization of their goods, therefore, the success of this industrial sector depends on the optimal management of its channels marketing and distribution.

Objective: To know the type of Information and 
Communication Technologies used in the marketing and distribution channels. Methodology: 27 companies registered in the National Chamber of Body Manufacturers (CANFAC) and 10 companies registered in the Tungurahua Production Directorate were taken into consideration, to develop the research the descriptive method was used through a survey of managers of the companies, which was validated with Cronbach's Alpha internal consistency index for ordinal variables, Kuder Richardson KR-20 for dichotomous questions and approval of the instrument by 5 judges, in this case, the Aiken $\mathrm{V}$ reliability coefficient was applied, The bibliographic and field research modality was used. Results: the industrial sector uses electronic means for its marketing channels, $68.9 \%$ prefer social networks and web pages, $79.1 \%$ use the direct channel to distribute goods, there is also little knowledge about the operation of the Enterprise Resource Planning (ERP) system and Customer Relationship Management (CRM) system. Conclusion: information and communication technologies have many alternatives, the same that are applied depending on the size that each one has, therefore, suggest alternatives that allow efficient management of the marketing and distribution channel between internal and external clients of the company.

\section{Introducción}

La cadena productiva consta de varias etapas las mismas que ayudan con la optimización de recursos, siendo una de las etapas más importantes los canales de comercialización y de distribución, debido a que, en estas fases, la empresa busca diseñar estrategias con las cuales se pretende cumplir con los objetivos de su plan estratégico en el cual se enmarca todas las acciones necesarias a seguir para cumplir con todas las metas económicas impuestas por la junta directiva, por lo tanto es de gran importancia el optimizar los canales de comunicación empresariales con el fin de cubrir con más facilidad las necesidades del cliente, en la actualidad existe una alta aceptación de las empresas que adoptan nuevas herramientas tecnológicas para todos sus departamentos. En la provincia de Tungurahua existe el sector industrial encargado de diseñar y producir carrocerías para autobuses que está constituido por 37 empresas que están conformadas por departamentos como: Finanzas, producción, marketing y ventas, el presente trabajo informará los resultados obtenidos sobre las adopciones de determinados recursos informáticos empleados en los canales mencionados. 


\section{Metodología}

Esta investigación se basa en el método descriptivo según añade el autor Narváez (2017), que dicho método permite emplear las herramientas con las cuales se pretende evaluar a un grupo de personas por lo tanto es importante aplicar cálculos estadísticos para obtener la población y muestra de cualquier proyecto de investigación, este proceso se lo realiza con el propósito de poder analizar el fenómeno investigativo, ya que estos cálculos son el resultado directo debido a que se emplea una proyección cuantitativa, la cual busca establecer los criterios necesarios para poder analizar los datos históricos por medio de teoremas matemáticos y así identificar un patrón de crecimiento que facilite la medición de su relación frente a este problema de investigación.

Según el autor Muñoz (2016), afirma que la modalidad aplicada en esta investigación es la de campo, porque es necesario el empleo de una herramienta de investigación como lo es la encuesta, la misma que permite extraer datos con los cuales se puede cuantificar los datos recopilados por los gerentes de las carrocerías de este sector industrial en la provincia de Tungurahua, es decir, este tipo de herramienta obligatoriamente deberá contar con un soporte estadístico para corroborar su validez y su viabilidad antes de aplicar el cuestionario sobre nuestro público objetivo.

Según el autor Franco (2017). considera que una las principales herramientas de gran utilidad para el desarrollo de cualquier tipo de investigación es el software llamado como IBM SPSS, el cual con sus múltiples herramientas permite al investigador tabular una gran cantidad de datos de una manera sencilla y con un alto grado de precisión, este tipo de software con el paso del tiempo fue evolucionando hasta llegar a la versión 25, la misma que permite trabajar con 2,5 millones de datos y más de 250 mil variables en todos sus formatos, es por ello que es muy conocido en el mundo de las ciencias sociales y comerciales y por su gran utilidad fue cambiando de acuerdo a las necesidades de los investigadores, su papel fundamental es la de dar validez a toda la información recopilada durante la aplicación de este cuestionario al sector carrocero de la provincia de Tungurahua.

De acuerdo con Aiken (2003), afirma que la V de Aiken es un proceso matemático con la cual se proporciona un valor numérico a cada una de las preguntas que conforman una encuesta, el cual es procesado por medio de una formula estadística, en donde el resultado obtenido puede ser 1 o 0 , y entre más se aproxime al número 1 mayor será la validez del instrumento investigativo, los datos para poder aplicar este coeficiente son obtenidos de una matriz proveniente de un juez miembro de un tribunal evaluador para así poder dar credibilidad a este tipo de encuestas.

Otro de los métodos para la validación de datos se da con la técnica de confiabilidad conocida como KR20, de acuerdo con el criterio de Smith (2021) establece que este 
método para validar la estructura interna de una encuesta, es empleado para la validación de las preguntas dicotómicas a las cuales se les asigna un valor para cada pregunta, al final se identificara cada uno de los ítems pertenecientes a la fórmula para obtener una calificación con la cual se establezca un índice con el cual se elabore de validez a una encuesta de investigación, el cual debe respetar la siguiente formula:

\section{Ecuación 1}

Formula KR20

$$
\mathbf{r}_{\mathbf{k r}_{20}}=\left(\frac{\mathbf{k}}{\mathbf{k}-\mathbf{1}}\right)\left(\mathbf{1}-\frac{\sum(\mathbf{p} * \mathbf{q})}{\boldsymbol{\sigma}^{\mathbf{2}}}\right)
$$

En donde:

$$
\begin{aligned}
& \mathrm{k}=\text { Número de ítems del instrumento } \\
& \mathrm{p}=\text { Porcentaje de personas que responde correctamente cada ítem } \\
& \mathrm{q}=\text { porcentaje de personas que responden incorrectamente cada ítem } \\
& \sigma^{2}=\text { Varianza total del instrumento }
\end{aligned}
$$

\section{Población y muestra}

El desarrollo de la siguiente investigación está conformado por las empresas que se dedican a producir carrocerías metálicas para lo cual CANFAC (2020) establece que contamos con 27 empresas registradas en la provincia de Tungurahua. Adicionalmente según considera el Directorio de Producción de Tungurahua (2019), se encuentran registradas 10 empresas carroceras que no aparecen en los registros de CANFAC debido a que ellos no cuentan con un volumen alto de ventas, por tanto, la población de empresas carroceras en la provincia es de 37 empresas que se dedican a esta actividad manufacturera.

Métodos de Validación de la Encuesta

\section{Tabla 1}

Alpha de Cronbach

\begin{tabular}{ccc}
\hline \multicolumn{3}{c}{ Estadísticas de fiabilidad } \\
\hline $\begin{array}{c}\text { Alfa de } \\
\text { Cronbach }\end{array}$ & $\begin{array}{c}\text { Alfa de Cronbach basada en } \\
\text { elementos estandarizados }\end{array}$ & N de elementos \\
\hline, 824 &, 872 & 37 \\
\hline
\end{tabular}

Elaborado por: Grupo Investigador 
Análisis: aplicadas las 37 encuestas al sector carrocero obtenemos que el índice general es de 0,824 a nivel global de la encuesta, también tenemos un 0,872 a nivel individual de las preguntas.

Interpretación: Monegal (1999) considera que el resultado obtenido puede ser entre 0 y 1, y que entre más se acerque al número 1 mayor será el índice de confiabilidad que posee una encuesta, este coeficiente es aplicable únicamente en las preguntas que posean escala de Likert en sus opciones de respuestas. Una vez tabuladas las encuestas, se dispone de un índice de fiabilidad de 0,824 a nivel global y de 0,872 a nivel individual de todas las preguntas que posee la encuesta, lo que provee la confiabilidad necesaria para ejecutar este trabajo de investigación.

\section{Ecuación 2}

Coeficiente de confiabilidad KR-20

$\begin{aligned} r_{k r_{20}} & =\left(\frac{k}{k-1}\right)\left(1-\frac{\sum(p * q)}{\sigma^{2}}\right) \\ r_{k r_{20}} & =0,89\end{aligned}$

Interpretación: González (2013), asegura que KR-20 es un coeficiente aplicado a preguntas dicotómicas de una encuesta, en la cual se obtendrán valores entre 1 y 0 , un resultado es más aceptable cuando más cerca del número 1 se encuentre. Una vez procesados los datos registrados en la encuesta se consiguió un valor de 0,89 lo cual indica un nivel de confianza alto.

\section{Ecuación 3}

Coeficiente de confiabilidad V de Aiken

$$
\begin{gathered}
\qquad V=\frac{S}{n *(c-1} \\
\text { V de Aiken promedio }=\quad 0,91
\end{gathered}
$$

Interpretación: Muñoz (2016), establece que el coeficiente de V de Aiken es un coeficiente estadístico en el cual se obtienen valores entre 1 y 0 , de tal forma que cuando el resultado se acerque más al número 1 es debido a que existe un mayor acuerdo entre los jueces que han evaluado una encuesta como instrumento de investigación. Para el presente estudio se obtuvo un valor promedio de 0,91 , lográndose un alto nivel de confiabilidad por parte de los jueces calificadores.

\section{Marco Teórico}

Las Tecnologías de la Información y Comunicación en la actualidad abarca todas las herramientas de tecnología que tiene como fin específico brindar una comunicación 
amplia en diferentes aspectos como difundir información de forma masiva a las diferentes audiencias dentro del entorno a través de transferencia de datos de alta relevancia para sectores productivos (Suárez, 2016).

Según Romero (2018) menciona que, existen diferentes características que resaltan a las Tecnologías de la Información y Comunicación:

Inmaterialidad: esta característica específica se enfoca en el manejo de toda la información a través de la internet las cuales se puede difundir a distintos lugares a nivel mundial.

Instantaneidad: esta característica se enfoca en que los TIC'S están relacionados directamente con la comunicación, y a través de esta se comparte información a sitios lejanos es decir dentro y fuera del país a nivel global en cuestión de segundos.

Interactividad: dentro de estas características se encuentra la interactividad esta quiere decir que siempre va a existir un intercambio de información entre las personas que usan la tecnología y un computador, el mismo que difundirá datos ingresados por los usuarios a las personas encargadas de diferentes programas.

Calidad audiovisual: los TIC'S permiten que cualquier tipo de imágenes, videos e información difundida sea con los más altos estándares de nitidez, conforme pasa el tiempo se va realizando cambios para mejorar el entendimiento del usuario.

Digitalización: esta se enfoca en mantener toda la información ya sea textual, sonidos, o imágenes puedan compartirse a través de redes telemáticas, las mismas que requieren de un software o un hardware.

Innovación: las TIC'S se encuentran em cambios de forma continua, es decir que siempre se actualiza y mejoran ciertos parámetros como calidad audiovisual o mayor alcance de la información a diferentes sitios del mundo.

\section{Aplicación de las TIC'S en la comercialización}

La aplicación de las TIC'S en la economía a nivel mundial ha transformado las micro, medianas y grandes empresas, la gestión de las empresas se enfocan en la aplicación de las herramientas de tecnología para asociarlas con los fines de cada negocio, como también se ha beneficiado el sector de salud y educación a través de una comunicación eficaz y eficiente (Cano, 2018). De acuerdo con Sánchez (2017), establece que las TIC’S en el sector empresarial han tenido un alto impacto positivo debido a que vincula con facilidad al mayor número de personas con este ámbito, por tanto las empresas han sido beneficiadas directamente a través de las características funcionales de los programas informáticos, tal es el caso de la velocidad que poseen para procesar datos ya que en segundos difunden información para sus usuarios, sin embargo las empresas que no están 
listas para realizar un cambio tecnológico a gran escala, debido a que muchas instituciones temen desparecer del mercado.

\section{Aplicación de las TIC'S en el sector industrial}

Añade Plaza (2018), que en la actualidad es de vital importancia fomentar el uso de las Tecnologías de la Información y Comunicación debido a que está incrementa la competitividad de las empresas, mejora procesos de productividad además que crea valor agregado tanto para clientes internos como externos, la implementación de las TIC'S direcciona a que la organización cambie su ambiente con enfoque tecnológico de forma radical en la manera en cómo maneja sus procesos en sectores industriales tanto en creación de nuevos productos y su estructura.

Según el Instituto Ecuatoriano de Estadísticas y Censos (INEC, 2015), el sector manufacturero conforme pasa el tiempo se ha convertido en una actividad de gran importancia para la población ecuatoriana, y se ha visto la necesidad de implementar en sus procesos de desarrollo organizacional herramientas que incrementen el nivel de competitividad y alcance del mercado al que están dirigidos sus productos.

\section{Herramientas informáticas de gestión empresarial}

Redes sociales. - Según Ron (2019), afirma que son los medios digitales empleados por un alto número de usuarios de todo el mundo, con el propósito de conformar comunidades en las cuales se comparten el mismo interés hacia determinados aspectos, en los cuales se pueda intercambiar datos de uso común, estos pueden ser imágenes o videos, los cuales son de agrado de los miembros que conforma dicha red social, las principales redes sociales son: Facebook. Twitter, YouTube, Instagram etc.

Microsoft Office.-- Según Peña (2017) considera que es un paquete de programas informáticos cuyo segmento de mercado se da principalmente en el ámbito empresarial y educativo, el cual está constituido por procesadores de texto como el programa Microsoft Word, hojas de cálculos conocidas como Microsoft Excel, procesador de presentaciones también llamado como Microsoft PowerPoint y sobre todo el software de control de actividades empresariales llamado Microsoft Proyect, este conjunto de programas poseen licencias de pago, la vida útil de este tipo de software dependerá del tipo de licencia que adquiera el consumidor, siendo el paquete 365, el más empleado en un sin número de áreas ya sea en el sector público o privado.

Sistemas Operativos.- Moreno y Ramos (2017) confirman que es el tipo de software que se ejecuta de manera primaria dentro de la arquitectura de un sistema informático, que por medio de este programa se controlan los recursos físicos y se los adapta para que puedan ejecutarse aplicaciones virtuales dentro de este entorno gráfico, además de esta tarea, un sistema operativo de encarga de ejecutar los controladores necesarios para que 
se ejecuten los diferente periféricos que emplea un computador para su funcionamiento, siendo una de sus actividades principales la asignación de recursos necesarios para la ejecución de determinados entornos virtuales como lo son los juegos de video, en el mercado informático, existen sistemas para cualquier tipo de necesidad, siendo Windows y Linux los más presentes dentro del ámbito computacional.

Software CRM. - Menciona el autor Polo (2016) considera que El software CRM cuyo significado en inglés es Customer Relationship Management lo que traducido al español significa programa informático para la gestión de la relación con el cliente, este tipo de plataformas informáticas provee las herramientas necesarias para el manejo de la información más sensible de una empresa.

Para Valle (2015) considera que los datos financieros y sobre todo ofrece el manejo sistematizado de la cartera de clientes que posee cada organización así como la información necesaria para contactar con sus principales proveedores, este tipo de software provee la interfaz gráfica para monitorear las ventas en tiempo real del equipo de ventas que conforma cada empresa por medio de la utilización de leads, la cual actualizara en tiempo real el número de ventas realizadas y su respectiva ruta de ventas realizadas en un determinado tiempo.

Software ERP.- Benavidez (2017) afirma que el software informático para la gestión de los recursos empresariales (ERP) cuyas siglas en ingles significan Enterprise Resource Planning, está diseñado como una plataforma que sirve para gestionar las actividades diarias de la empresa tal es el caso del manejo de los registros contables, así como el despacho de pedidos y sobre todo el control de todas las operaciones que requiere la empresa para continuar su funcionamiento.

Según establece el criterio de Puerta y Valle (2016) consideran que este tipo de software ofrece las herramientas matemáticas con el fin de predecir los resultados de las operaciones financieras necesarias para la toma de decisiones sobre la ejecución de un determinado proyecto, esto se debe a que su estructura interna de ejecución permite el intercambio de datos entre flujos de funcionamiento interno para así poder analizar cada línea de negocios posee cada organización en un determinado tiempo, ya que los datos proporcionados por esta plataforma poseen un alto grado de confiabilidad .

Diferencias entre el software CRM y ERP. - Para Vidal (2005) considera que la plataforma CRM se enfoca en gestionar las relaciones que la institución posee con sus clientes, así como el monitoreo de su fuerza de ventas, en cambio la plataforma ERP se encarga gestionar y también controlar los procesos de funcionamiento de la empresa.

Según Smith (2014) analiza que la plataforma CRM se caracteriza por ser un software Front Office debido a que se pueden diseñar estrategias de ventas con los clientes y la 
logística necesaria para el control de los procesos externos para la gestión de la organización, por otro lado, la plataforma ERP facilita la planeación de los procedimientos internos de funcionamiento de la empresa debido a que este tipo de programa informático es back office y no interfiere en las relaciones de la organización con el cliente y más bien se centra en todos los procesos necesarios para mantener el funcionamiento interno de la empresa.

Para Swift (2015) afirma que, en cuanto a costos de implementación, el costo accesible que posee el software CRM debido a que su estructura de funcionamiento es más reducida ya que controla menos áreas de cada empresa y además poseen software de licencia gratuita lo que ayuda a la selección de un amplio número de opciones, por otro lado, se encuentra la plataforma ERP en cambio esta es más costosa de implementar debido a que cuenta con los servicios en la nube y es necesario el proporcionar mantenimiento periódico para garantizar la integridad de sus datos.

Según Duer (2003) considera que, los módulos que constituyen este tipo de software es su, en el caso del programa CRM posee módulos para marketing, gestión comercial y los servicios para relacionarse con el cliente como lo son la atención y servicios de postventa, el caso del software ERP incorpora módulos para poder manipular a los movimientos financieros, el departamento de producción, un control de los inventarios, además de ofrecer un monitoreo constante de los movimientos contables, ya que su propósito principal es controlar la logística necesaria para las gestiones que requiere la empresa para su funcionamiento.

Afirma Valencia (2003) que para implementar estas plataformas informáticas es necesario conocer todas las exigencias para instalar y activar estos sistemas, en el caso del programa informático CRM, el cual se caracteriza por poseer un método fácil de instalar debido a que no está presente en todos los departamentos que posee la empresa, en el otro extremo se encuentra la plataforma ERP, la cual requiere contar con un personal lo suficientemente capacitado para poder manipular e instalar adecuadamente ya que este sistema informático se encuentra presente en todos los departamentos de la empresa lo que provoca que su instalación sea más larga debido a que es necesario el verificar todos los procesos requeridos para su funcionamiento.

\section{Los canales de comercialización y distribución del sector manufacturero}

Los canales de comercialización son los medios utilizados por las empresas con el propósito de comunicar, mediante mensajes para convencer a los consumidores sobre todos beneficios que ofrece un determinado bien, tomando en cuenta los gustos y preferencias que posee cada consumidor en un determinado segmento de mercado (Valencia, 2017). 
Los tipos de canales de comercialización son los físicos y digitales, siendo los medios físicos conocidos como los medios más tradicionales para la venta de bienes ya que es necesario contar con un punto de venta físico, por otra parte, los medios digitales de venta se caracterizan por emplear la tecnología como los principales medios para comercializar sus bienes, la cual se encarga de crear canales automatizados para conseguir dicho fin (Celaya, 2008).

De acuerdo con el criterio de Acosta (2017) existen diferentes tipos de canales de comercialización como a continuación se detalla:

Canales tradicionales: este tipo de canal se dedica netamente a expender productos en su mayoría de primera necesidad y se encuentra al alcance del mayor número de clientes posible, por lo tanto, no es importante el uso de la tecnología porque estos se encuentran en lugares cercanos como tiendas, mercados y kioscos en donde las personas acuden a estos espacios con el fin de socializar.

Canales automatizados: se caracteriza por ofertar los productos a través de máquinas electrónicas, debido a que están vinculadas directamente con la tecnología ya que no necesitan de una persona para la venta de productos, sino que esta cumple sus funciones de forma en como la programen.

Canales audiovisuales: este canal tiene diferentes medios por el cual comercializa un producto o servicio como la publicidad a través de radio y televisión.

Canales electrónicos: para que este tipo de canal funcione es necesario el internet y contar una base de datos de los clientes potenciales por el cual a través del correo previamente descrito con información de los productos se pueda llegar al consumidor.

\section{Canales de distribución}

Según Paz (2010) menciona que los canales de distribución son el conjunto de acciones que emplean diferentes entidades o personas con el fin de que un cierto producto llegue hasta el final de la cadena que es el consumidor final, en los cuales se encuentran inmersos participantes primarios y especializados.

De acuerdo con el criterio de Cruelles (2016). Analiza que los canales de distribución son los todos los medios físicos empleados por una institución con el propósito de poder transportar sus bienes para que estos puedan ser llegar con el consumidor final de una forma más rápida, estos medios empleados varían dependiendo de la naturaleza del bien y las necesidades que requiere la empresa

El autor West (2007) menciona que, los tipos de canales de distribución son empleados para que un bien comercial llegue a su destino y se clasifica en: 
Canal directo: este tipo de canal es el más completo porque requiere de espacios físicos para almacenar los productos como también de transporte para hacer llegar el producto a las manos del cliente, por lo general este tipo de canal lo usan las fábricas la cual tiene un conjunto de personas que pertenecen a la empresa productora los cuales se encargan de vender los productos directamente al consumidor final, es decir en esta línea no participan intermediarios, la relación es directa, empresa-cliente

Canal corto: este se encarga de distribuir los productos de un fabricante a minorista o detallista esto quiere decir los pequeños negocios que se dedican a la compra y venta de artículos en pocas cantidades hasta llegar al consumidor final.

Canal indirecto: en este tipo de canal existen más de cuatro intermediarios que extienden los productos, los mismos que son comercializados, pero con costos un poco más altos debido a que la cadena de distribución la definen de acuerdo con sus necesidades.

\section{Sector carrocero en la provincia de Tungurahua}

De acuerdo con el autor el diario La Hora (2018), la provincia de Tungurahua se caracteriza por poseer múltiples actividades económicas de gran importancia para el país estas son el sector agropecuario, el calzado, la maquila de varias prendas de vestir y sobre todo el sector metalmecánico, el mismo que posee un número significativo de empresas que se dedican a la manufactura de carrocerías de auto buses con el fin de satisfacer a la demanda nacional por medio de sus diseños que van de la mano con las normas de seguridad impuestas por el INEN, con el propósito de proveer las normas de seguridad necesarias para dotarles de un valor adicional a este tipo de vehículos frente a sus competidores nacionales e internacionales, debido a las políticas implementadas por los gobiernos centrales, muchas empresas desaparecieron ya que no contaban con los recursos necesarios para poder enfrentar a las condiciones cambiantes del mercado de autobuses en el Ecuador. De acuerdo con la Cámara Nacional de Fabricantes de Carrocerías (CANFAC, 2020), establece que el total de empresas que se dedican a la actividad manufacturera de autobuses son 27 , y se encuentran distribuidas de la siguiente forma: 26 en el Cantón Ambato y 1 en la ciudad de Pelileo. Así mismo es necesario indicar que existen 10 empresas carroceras repartidas, que se ubican 7 en el cantón Ambato, 1 en el cantón Baños de agua santa y 2 en Tisaleo, estas no se encuentran registradas en CANFAC. Estas instituciones contribuyen al desarrollo económico del país generando fuentes de empleo para la provincia de Tungurahua, además de aportar con el 13,7 \% al PIB nacional, y anualmente con cientos de miles de dólares en impuestos para el gobierno ecuatoriano, este sector industrial no cuenta con medidas administrativas para proteger a este tipo de industria frente a los bienes importados. 


\section{Resultados}

\section{Figura 1}

Preferencia de Canales de comercialización

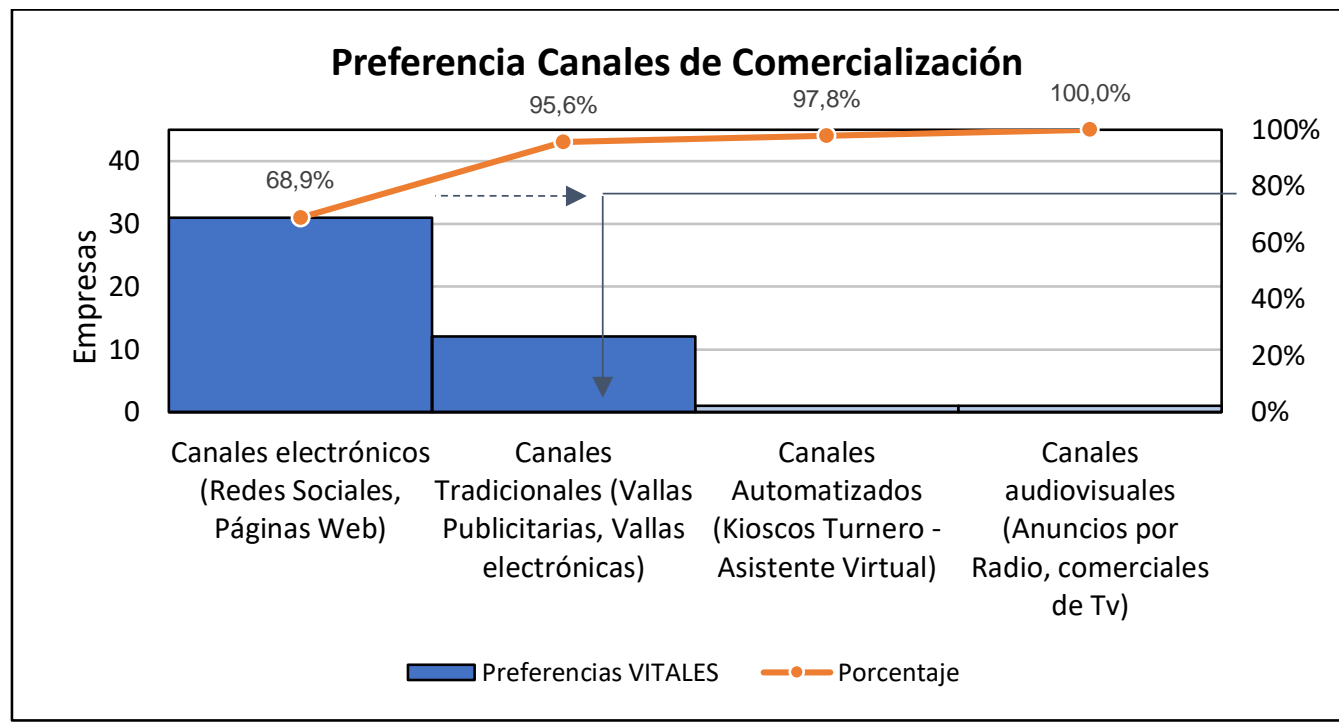

Nota: En el Pareto se observa la preferencia hacia los Canales de Comercialización por parte de las 37 empresas del sector carrocero.

Fuente: Elaboración propia

Debido a su gran versatilidad, se tiene con un claro ganador a los canales electrónicos, las empresas carroceras en su mayoría implementan herramientas con las cuales se pueda evaluar el nivel de satisfacción de un cliente tal es el caso de la encuesta, una muy potente herramienta estadística que puede ser difundida en redes sociales o páginas web, y con la que se puede determinar entre otras cosas, factores para mejorar la comunicación entre la empresa y el consumidor. En segundo lugar, tenemos a los Canales Tradicionales de comunicación, son mensajes difundidos por los medios de radio y televisión, así como las vallas publicitarias y las electrónicas, aunque por su naturaleza no ofrecen fácilmente, datos que faciliten la retroalimentación de la información adquirida, por tanto, estas herramientas han perdido su fuerza de impacto en el consumidor, siendo su porcentaje acumulado el 95,6\%. 


\section{Figura 2}

Preferencias de Canales de distribución

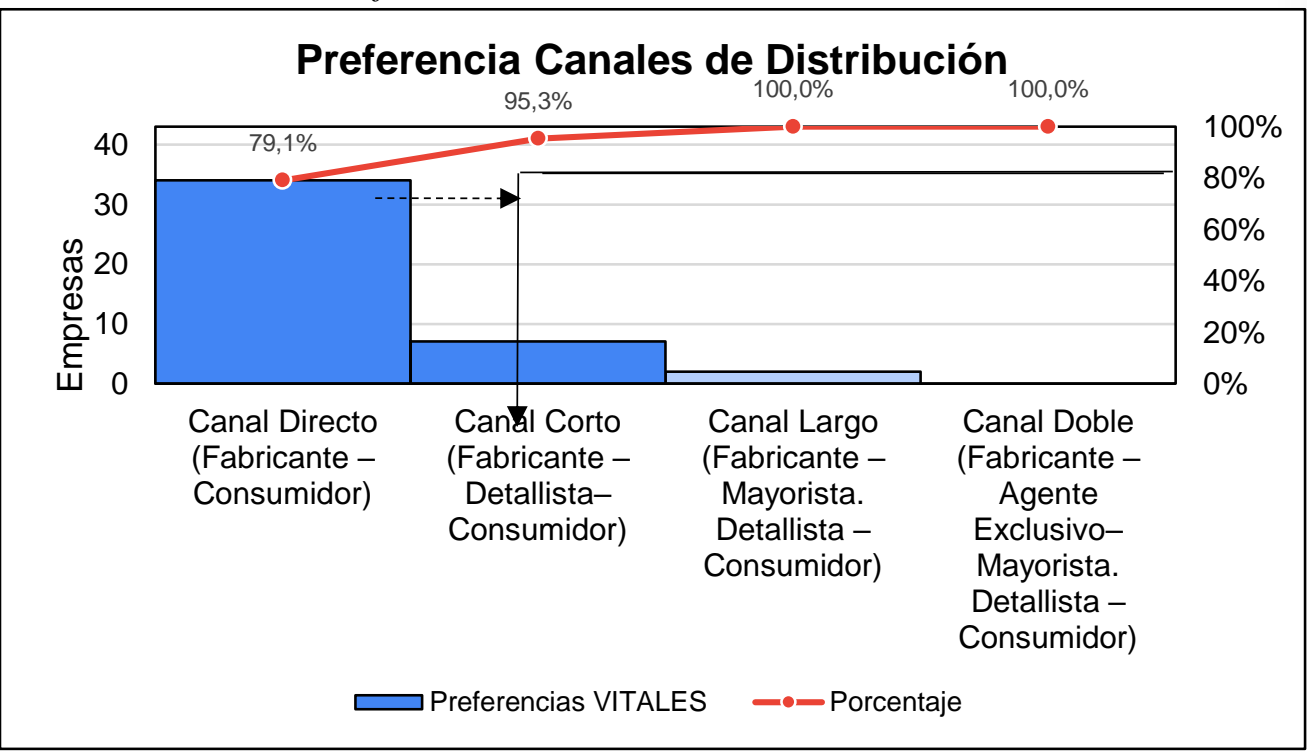

Nota: Se aprecia la preferencia del canal de Distribución en este sector industrial se destaca el canal directo.

Fuente: Elaboración propia

El sector industrial, en primer lugar, se utiliza el Canal Directo de Distribución, en el que no existe la participación de intermediarios en su comercialización, es decir que el cliente se contacta directamente con la empresa para adquirir este tipo de bienes. En un segundo lugar y con un bajo porcentaje $(15,4 \%)$, se encuentra el uso del Canal Corto, con la influencia de terceras personas que intervienen en el desarrollo de la venta, por un cierto porcentaje como comisión ganada, restándole beneficios a la empresa.

Figura 3

Las TIC'S como medida de control en canales de comercialización y distribución

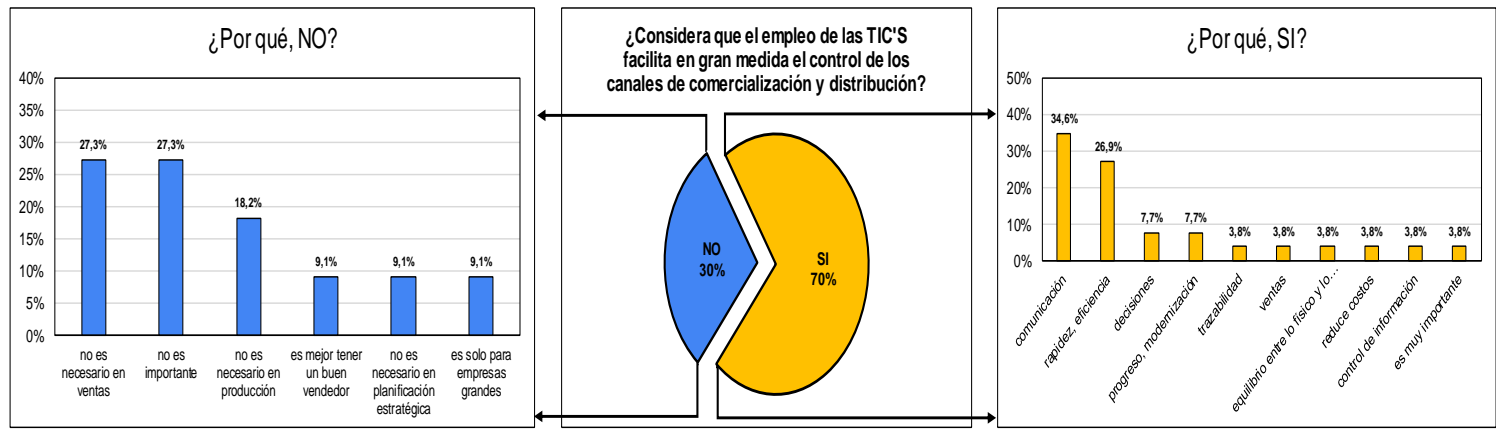

Nota: se observa que el 70\% consideran importante la aplicación de las TIC'S en los canales de comercialización y de distribución, mientras que el $30 \%$ afirman que no es importante.

Fuente: Elaboración propia 


\section{www.cienciadigital.org}

El $70 \%$ de los encuestados nos da una respuesta afirmativa, ellos consideran que el empleo de las TIC'S benefician a las empresas para controlar sus canales de comercialización y distribución; con estas herramientas se puede gestionar de forma eficiente la comunicación entre los clientes internos y externos. Mientas que, el $30 \%$ de empresas que se manifestaron negativamente, en su mayoría consideran que estas tecnologías no son necesarias para las ventas, tampoco influyen con los procesos de producción, varios empresarios estiman que su aplicación solo debería darse en empresas grandes.

\section{Figura 4}

\section{Beneficios del uso de Software ERP}

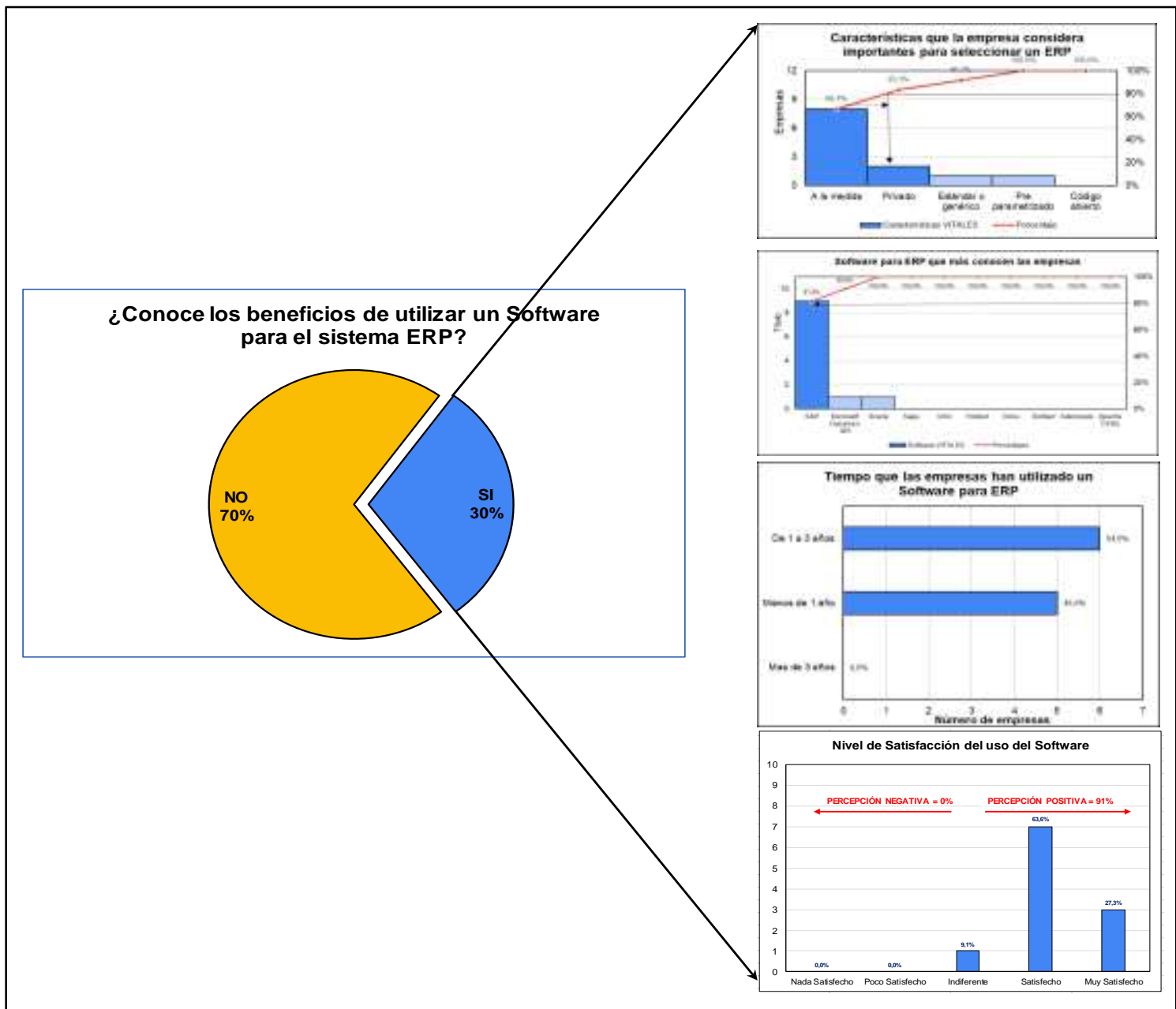

Nota: Se observa un $70 \%$ que poseen desconocimiento del software para el sistema informático para la Gestión de los Recursos Empresariales (ERP).

Fuente: Elaboración propia

La gran mayoría de los empresarios encuestados, es decir el $70 \%$ de las empresas "desconocen" los beneficios que integran este tipo de plataformas informáticas. El 30\% 


\section{www.cienciadigital.org}

de la población, afirma que el software más empleado en este campo es el SAP porque posee los módulos necesarios para poder acoplarse a la necesidad de cada departamento de la empresa, en su mayoría las empresas encuestadas afirman que acogieron este sistema y lo implementaron por un lapso menor a los 3 años, los cuales se encuentran satisfechos con el desempeño, esto nos lleva a reconocer que la industria carrocera tiene una gran oportunidad de desarrollo con estos sistemas informáticos.

\section{Figura 5}

Beneficios del Software para el manejo de la Gestión de las relaciones con los clientes (CRM)

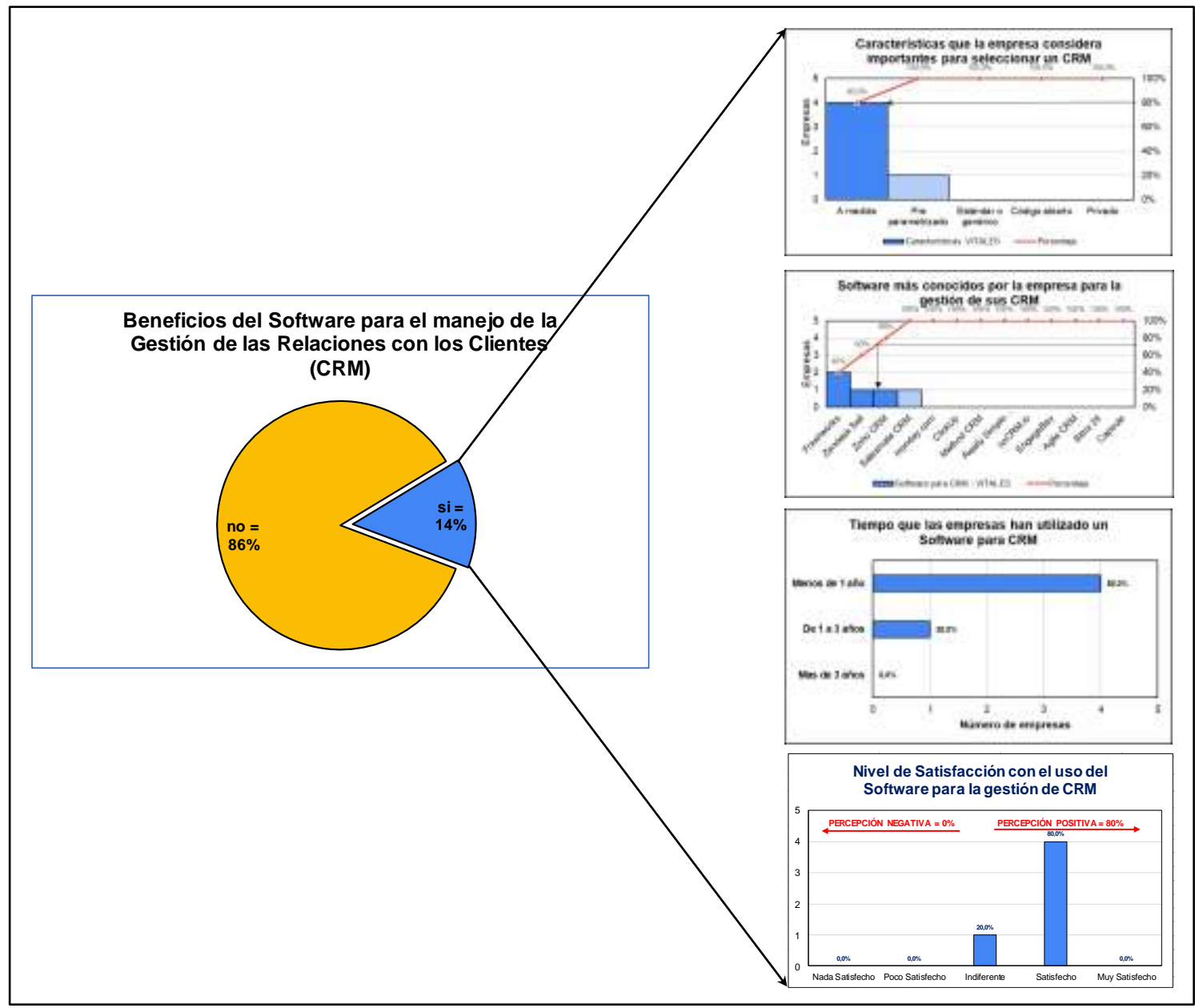

Nota: Se establece que el $86 \%$ de la población encuestada desconoce el sistema para la Gestión de Relaciones con el Cliente (CRM).

Fuente: Elaboración propia

De los resultados obtenidos, se analiza que el $86 \%$ afirma no conocer sobre todas las características funcionales que posee este tipo de software. El 14\%, que manejan el sistema CRM, indican que la principal característica para seleccionar estos programas son los parámetros para el diseño de su funcionamiento, como lo es el tamaño de la empresa, 
así como el volumen de ventas, las cuales son factores necesarios para programar la estructura modular de este sistema, siendo el más empleado el software Freshworks, Zendesk Sell y Zoho CRM. el sector carrocero puede mejorar sus procesos administrativos significativamente, adoptando estas tecnologías.

\section{Figura 6}

Importancia de las TIC'S en el manejo de la cadena de suministros

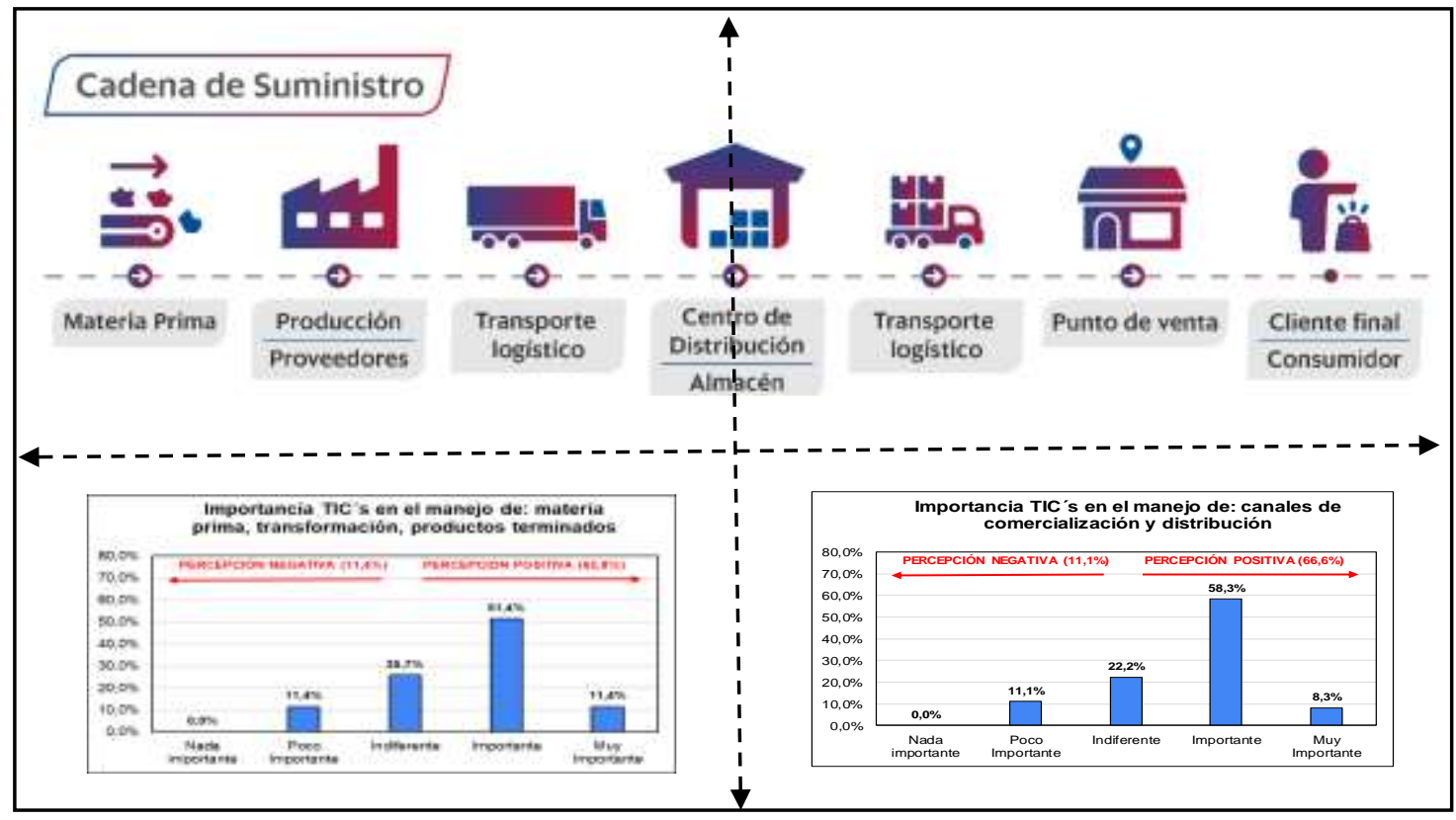

Nota: El 68\% de la población afirma que es importante la aplicación de las TIC'S en la cadena de suministro.

Fuente: Elaboración propia

Se aprecia una percepción positiva del $66,6 \%$ calificando entre importante y muy importante, la implementación de las TIC'S en los canales de comercialización y distribución. También se observa que existe una percepción positiva entre importante y muy importante del 62,8\% en el manejo de materia prima, transformación (producción) y producto terminado. Esto quiere decir que, en opinión de la industria carrocera de la provincia de Tungurahua, la tecnología influye positivamente en los procesos internos y externos que requiere la empresa para su funcionamiento.

\section{Discusión}

El desarrollo investigativo del presente trabajo investigativo utilizó a las 37 empresas productoras de carrocerías metálicas para autobuses ubicadas en la provincia de Tungurahua, la cual busca conocer la aplicación de las TIC'S en dicho sector, para lo cual el autor Pérez (2015) establece que las herramientas informáticas son integradas en los canales de comercialización, ya que pueden ser empleados para la difusión de mensajes tanto para los colaboradores internos y externos de la empresa. Debido a que se mantiene 
un canal de comunicación directo entre el personal operativo, administrativo, los clientes y proveedores, este tipo de contacto se lo puede realizar por medio de video llamadas, redes sociales o salas de chats especializadas en los intereses que posee la empresa, además estas herramientas poseen funciones en las cuales se pueden implementar herramientas para evaluar una determinado aspecto, de acuerdo con la autora Quijada (2020) afirma que, las herramientas informáticas son de fácil manipulación debido a que la interfaz de funcionamiento se acopla con las necesidades de los usuarios, los cuales además de servir como canales de comunicación internos, también se pueden convertir en canales interactivos de comunicación externos ya que los nuevos autores de este tipo de comunicación son los proveedores y clientes. En este sentido se considera que el 68,9\% de las empresas carroceras de la provincia de Tungurahua están conscientes de todos los beneficios que las TIC'S poseen por tanto las aplican dentro de su funcionamiento ya que les permite monitorear aquellos aspectos con el claro objetivo de mejorar su posicionamiento de marca. En sentido, el $70 \%$ de los empresarios consideran que el empleo de estas herramientas se da por las características que estas poseen, siendo la comunicación uno de los factores más importantes para seleccionar este tipo de medios para la gestión de los canales de comercialización de la empresa esto se debe a las herramientas que posee para la manipulación de ciertas funcionalidades para evaluar los canales que cada institución posee para sus actividades.

El canal más empleado en sector carrocero es el canal directo es decir aquel canal en el cual interviene la empresa y el cliente final, de acuerdo con el autor Cruz Roche (2017) considera que los canales de comunicación directos son aquellos en los cuales los principales participantes son el productos y el consumidor final, es decir que existe un canal de comunicación de frente entre las dos partes, debido a que no existen intermediarios para estas operaciones comerciales, esto se debe a que en estos tiempos actuales, en los cuales existe gran presencia del uso de los dispositivos electrónicos los cuales amplían la cantidad de actividades que puede realizar el departamento de marketing de una institución, este tipo de acciones están siendo implementadas por el sector carrocero de la provincia de Tungurahua en un 79,1\%, ya que consideran que los productos y sobre todo la imagen de su empresa es más fácil de manejar desde un computador o cualquier dispositivo electrónico con el cual e ofrezca un canal de comunicación directo entre estas empresas manufactureras y los consumidores finales de este tipo de bienes.

Otro de los datos más interesantes que arrojo este tipo de estudio se da con la aplicación del software ERP, de acuerdo con los autores Valle y Puerta (2017) afirman que sistema informático está conformado por siglas en el idioma inglés cuyo significado al idioma español es Planificación de recursos empresariales posee un propósito principal, el cual es dar una mayor facilidad para manipular la información de una manera centralizada para lo cual es necesario controlar los métodos de entrada y salida de las áreas como marketing, 
servicios, atención al cliente inventarios y sobre todo la logística interna y externa que posee la empresa. La industria manufacturera de autobuses de la provincia de Tungurahua aplica en menor escala este tipo de sistemas siendo que el $30 \%$ de las empresas que emplean este tipo de sistemas, de las cuales, el $66 \%$ considera que es fundamental su aplicación debido a que se puede implementar en base a las necesidades que posee cada empresa, estas empresas consideran que el sistema SAP es aquel programa más empleado por este sector industrial, cuya implementación dentro de las operaciones internas se da en un tiempo menor a los 3 años, siendo la percepción positiva del $91 \%$ respecto a este tipo de softwares informáticos, este tipo de sistemas poseen múltiples características las cuales atraen a determinados segmentos de mercados.

Otro aspecto de gran importancia se da en la aplicación del Software para la gestión de las relaciones con los clientes (CRM), de acuerdo con el autor Valle (2017), Afirma que este tipo de sistemas se caracteriza por ofrecer herramientas para manipular en tiempo real aquellos datos ingresados por los clientes con el propósito de emplearlos para desarrollar e implementar estrategias con el fin de poder identificar oportunidades de crecimiento para este sector productivo. La encuesta aplicada a este segmento productivo arroja que software CRM también llamado como el cuadro de mando integral es implementado únicamente por el $14 \%$ de las empresas existentes en la provincia de Tungurahua, las cuales seleccionan este tipo de programas informáticos debido a la facilidad que ofrecen para configurarlos ya que el $80 \%$ lo elige para modificarlo en base a sus necesidades, siento que el $80 \%$ se atrevió a implementarlo debido a determinados parámetros que requieren cubrir con las funcionalidades que estos sistemas poseen, El programa Freshworks es el que más presencia posee con un $40 \%$ del total de empresas que pertenecen al sector carrocero.

\section{Conclusiones}

- El 68,9\% prefiere los canales de comercialización preferidos por el sector carrocero en la provincia de Tungurahua se da por los canales electrónicos compuestos principalmente por redes sociales y páginas web.

- El canal directo ocupa el 79,1\% de aceptación dentro del sector carrocero debido a que es el principal medio con mayor utilización dentro de los canales de distribución funcionan con el principal medio para difundir comunicados y promociones para atraer a nuevos clientes.

- Los beneficios de las TIC'S poseen un alto grado de aceptación lo cual es evidente en un grado de aceptación del $70 \%$ de las empresas encuestadas, las cuales consideran que uno de los principales factores que facilitan la implementación de estas tecnologías es la rapidez para conseguir canales seguros y eficientes para la comunicación entre los departamentos de la empresa. 
- Se considera que un $30 \%$ de las empresas carroceras conocen los beneficios que poseen el sistema para la planificación de recursos empresariales (ERP) el cual es usado debido a que cuenta con varios parámetros con los cuales se puede aplicar estos sistemas en base a las necesidades de la empresa, el sistema más conocido es el SAP con un $80 \%$ de presencia en las empresas Tungurahuenses.

- El 14\% de instituciones encuestadas concuerdan que los beneficios del software para el manejo de la gestión de las relaciones con los clientes (CRM) son amplias en base a las funcionalidades que poseen estas herramientas informáticas con el procesamiento en tiempo real de todos los requerimientos que esta empresa posee con sus clientes, siendo el sistema Freshworks el más empleado.

- Las empresas en el sector carrocero utilizan softwares comunes, deberían utilizar softwares más especializados como el CRM o el ERP. El software ERP que podría ser empleado para empresas pequeñas es el Soft Doit, en el caso de las empresas medianas podrían utilizar el sistema Wolters Kluwer, mientras que se puede emplear el software SAP proveniente de la firma Systemanalyse Programmentwicklung para empresas grandes. En el caso del sistema CRM para empresas pequeñas es el HubSpot CRM para empresas pequeñas, el sistema CRM ágil para empresas medianas y el sistema Freshworks para empresas grandes.

\section{Referencias Bibliográficas}

Acosta, A. (2017). Canales de distribución. Bogotá: Fondo editorial Areandino.

Aiken, L. (2003). Tests Psicológicos y Evaluación. México: Pearson Educación.

Benavidez, M. (2017). Impacto de las Tics en la empresa. Mexico: Ediciones Universitarias.

CANFAC. (23 de septiembre de 2020). Obtenido de http://www.canfacecuador.com

Cano, G. (2018). Las Tics en las empresas Evolución de la Tecnología y cambio estructural en las organizaciones. Dominio de las Ciencias, 12.

Celaya, J. (2008). La empresa en la WEB 2.9. Madrid - España: Editorial Grupo Planeta.

Cruelles Ruiz, J. A. (2016). Productividad e Incentivos. Mexico: Marcombo Ediciones Tecnicas.

Cruz Roche, I. (2017). Canales de distribucion en la empresa. Madrid - España: Grupo Anaya. 
Dirección de producción de Tungurahua. (16 de abril de 2019). Obtenido de https://rrnn.tungurahua.gob.ec/promocion/productos/get_by_categoria/5616823e 4def93a404000029

Duer, W. (2003). CRM: Customer Relationship Management: qué es, para qué sirve y cómo se implementa un proyecto de tecnología al servicio de la atención al cliente. En W. Duer. Mexico: Mp Ediciones Corporation.

ENI. (2017). Ofimatica Profesional. Madrid: Eni Ediciones.

Franco, A. (2017). Herramientas para procesar informacion. Mexico: Pearson Educacion.

González, L. (2013). Estadistica Descriptiva y Probailidad. Bogota: Editorial Coordinacion Universitaria.

ICB. (2020). Comunicacion Oral y Escrita en la empresa. Malaga - España: ICB Editorial.

INEC. (5 de septiembre de 2015). www.ecuadorencifras.gob.ec. Obtenido de www.ecuadorencifras.gob.ec:

https://www.ecuadorencifras.gob.ec/documentos/webinec/Estadisticas_Economicas/Tecnologia_Inform_Comun_Empresastics/2015/2015_TICEMPRESAS_PRESENTACION.pdf

La Hora. (24 de febrero de 2018). La hora. Obtenido de https://lahora.com.ec/tungurahua/noticia/1102138004/sector-carrocero-detungurahua-mejoro-en-los-ultimos-cinco-meses

Monegal, M. (1999). Introduccion al SPSS: Manipulacion de datos y Estadistica Descriptiva. Barcelona. España: Edicions de la iniversit at Barcelona.

Moreno, J., \& Ramos, A. (2017). Sistemas Operativos y Aplicaciones Informáticas. Madrid - España: RA-MA S.A.

Muñoz, C. (2016). Como elaborar y asesorar una investigacion de tesis. Mexico DF: Pearson Education.

Narvaez, P. (2017). Metodologia de la investigacion cientifica y bioestadistica. Santiago de Chile: Masters RIL.

Paz, H. (2010). Canales de distribución: gestión comercial y logística. Buenos Aires: Lectorum. 


\section{www.cienciadigital.org}

Perez, R. d. (2015). Gestion de empresas de comunicacion. Mexico: Comunicacion Social, Ediciones y Comunicaciones.

Plaza, I. (2018). Calidad en Actividades de I+D+i. Aplicacion en el sector TIC. Madrid España: Libros RJ.

Polo, P. (2016). Gestion Adminsitrativa. Bogota: Ediciones Educativas.

Romero, V. (2018). Metodologías y tecnologías de la información en la educación. Mexico: Area de Innovacion y Desarrollo.

Ron, R. (2019). Niños, Adolecentes y Redes Sociales. Madrid - España: EDISIC.

Sanchez, M. (2017). Investigaciones Cualitaticas en Clencia y Tecnologia. Mexico: CICTEC.

Smith, A. (2014). La estrategia CRM. En A. Smith. Mexico: en50minutos.es.

Smith, S. (28 de abril de 2021). Platinum Educational Gruop. Obtenido de https://platinumed.zendesk.com/hc/en-us/articles/214600006-What-is-KR20-

Suárez, R. (2016). Tecnologías de la información y la comunicación: Introducción a los sistemas de Información y de Telecomunicación. España: Ideaspropias Editorial S.L.

Swift, R. (2015). En R. Swift, CRM: coo mejorar las relaciones con los clientes (pág. 34). Mexico: Pretenci Hall.

Valencia, I. (2003). CRM, Gestion de la Relacion con los clientes. En I. Valencia, CRM, Gestion de la Relacion con los clientes (pág. 86). Madrid: FC Editorial.

Valencia, M. (2017). Posicionamiento de Marca y su influencia en la gestion administrativa. Bogota: UDM.

Valle, A. (2015). Estrategias para el Uso de un CRM. Mexico: It Campus Academy.

Valle, A. (2017). Estrategias para el uso de un CRM. Mexico: Ediciones Educativas.

Valle, A., \& Puerta, A. (2016). Consultoria en Tics. En A. Valle, \& A. Puerta, Consultaria en Tis, Gestion del sofdtware ERP y CRM (pág. 76). Mexico: IT Campus Academy.

Valle, A., Puerta, A., \& Nuñez, R. (2017). Curso de Consultoria TIC, Gestion, Software ERP y CRM. Vigo - España: IT Campus Academy. 


\section{www.cienciadigital.org}

Vidal, I. (2005). Como conquistar el mercado con una estrategia CRM. En I. Vidal. Madrid: FC Editorial.

West, A. (2007). Gestión de la distribución comercial. Madrid: Díaz de Santos.

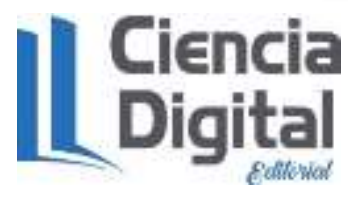


El artículo que se publica es de exclusiva responsabilidad de los autores y no necesariamente reflejan el pensamiento de la Revista Ciencia Digital.

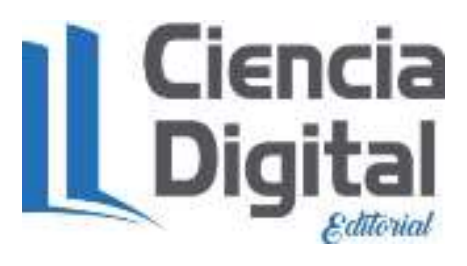

El artículo queda en propiedad de la revista y, por tanto, su publicación parcial y/o total en otro medio tiene que ser autorizado por el director de la Revista Ciencia Digital.
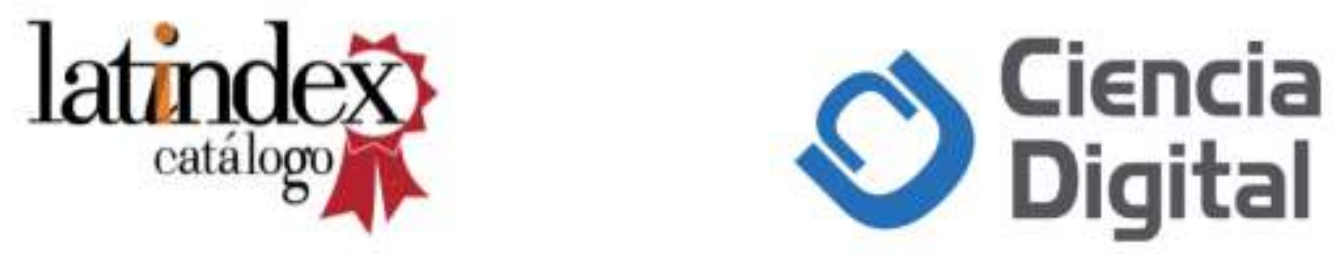

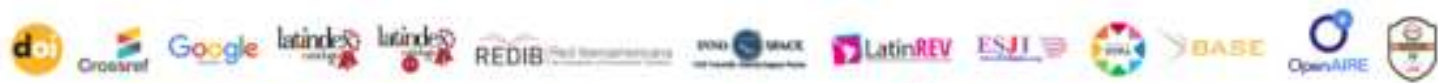

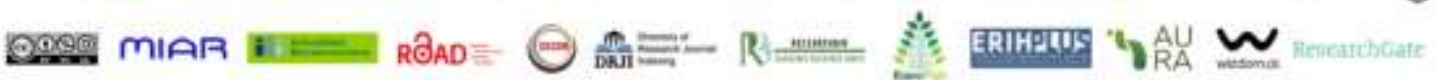

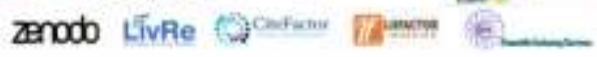

